\title{
Dispersion and nonlinearity compensation by spectral phase conjugation
}

\author{
Mankei Tsang and Demetri Psaltis \\ Department of Electrical Engineering, California Institute of Technology, Pasadena, California 91125
}

Received April 3, 2003

\begin{abstract}
We propose the use of spectral phase conjugation to compensate for dispersion of all orders, self-phase modulation, and self-steepening of an optical pulse in a fiber. Although this method cannot compensate for loss and intrapulse Raman scattering, it is superior to the previously suggested midway temporal phase conjugation method if high-order dispersion is a main source of distortion. The reshaping performance of our proposed scheme and a combined temporal and spectral phase conjugation scheme in the presence of uncompensated effects is studied numerically. (C) 2003 Optical Society of America
\end{abstract}

OCIS codes: $\quad 060.4370,060.7140,190.5040$.

Temporal phase conjugation (TPC) was proposed to compensate for group-velocity dispersion, ${ }^{1}$ selfmodulation, ${ }^{2}$ and intrapulse Raman scattering ${ }^{3}$ of an optical pulse in a fiber. However, when the pulse width is sufficiently short or the center wavelength is near the zero-dispersion point, third-order dispersion and self-steepening effects become more prominent and limit the reshaping performance of TPC. To compensate for the high-order effects, alternative methods $^{4-7}$ have been suggested, but many of them are either too complicated or are able to compensate for only a limited number of propagation effects. An interesting scheme that compensates for all effects by both TPC and a suitably chosen dispersion map was proposed by Pina et al. ${ }^{8}$

Instead of only conjugating the time envelope of an optical pulse midway through a fiber as in the TPC scheme, we propose to perform midway phase conjugation as well as time reversal of the pulse envelope (Fig. 1). With this method dispersion of all orders, self-phase modulation, and self-steepening in a fiber are automatically compensated. Conjugation and time reversal of an envelope combined are equivalent to conjugation of the optical pulse in the frequency domain, hence the name spectral phase conjugation (SPC). Consider a pulse $E(t)=A(t) \exp \left(-j \omega_{0} t\right)$ with envelope $A(t)$ and center frequency $\omega_{0}$. If we take the conjugation of the Fourier transform of $E(t)$, it becomes

$$
\begin{aligned}
\tilde{E}^{*}(\omega) & =\left[\int_{-\infty}^{\infty} A(t) \exp \left(-j \omega_{0} t\right) \exp (j \omega t) \mathrm{d} t\right]^{*} \\
& =\int_{-\infty}^{\infty} A^{*}(-t) \exp \left(-j \omega_{0} t\right) \exp (j \omega t) \mathrm{d} t,
\end{aligned}
$$

where the substitution $t \rightarrow-t$ is made. Hence conjugation of individual spectral components of a pulse is equivalent to phase conjugation and time reversal of the temporal envelope. TPC, on the other hand, corresponds to conjugation and inversion in the frequency domain.

Midway SPC is unique in the sense that it can compensate for all dispersion and most nonlinearities simultaneously. Consider the equation for general pulse propagation in a fiber,

$$
\frac{\partial A(z, T)}{\partial z}=\left\{\hat{D}_{T}+\hat{N}_{T}[A(z, T)]\right\} A(z, T),
$$

where $z$ is the propagation distance, $T$ is the retarded time with respect to the group velocity $1 / \beta_{1}$ of the pulse ( $\left.T=t-\beta_{1} z\right)$, and $A(z, T)$ is the pulse envelope. $\hat{D}_{T}$ is the linear operator,

$$
\hat{D}_{T}=-\frac{\alpha}{2}+\sum_{n=2}^{\infty} j \frac{\beta_{n}}{n !}\left(j \frac{\partial}{\partial T}\right)^{n}
$$

where the first term on the right-hand side is the loss term and the remaining terms are $n$ th-order dispersion terms. $\hat{N}_{T}$ is the nonlinear operator, which can be expressed as follows for the femtosecond pulse:

$$
\begin{aligned}
\hat{N}_{T}(A)= & j \gamma\left[|A|^{2}+\frac{j}{\omega_{0}} \frac{1}{A} \frac{\partial}{\partial T}\left(|A|^{2} A\right)\right. \\
& \left.-T_{R} \frac{\partial|A|^{2}}{\partial T}\right],
\end{aligned}
$$

where the first term on the right-hand side is the self-phase modulation term, the second term is self-steepening, and the third term is intrapulse Raman scattering. ${ }^{9}$ The subscript $T$ of $\hat{D}_{T}$ and $\hat{N}_{T}$ denotes the derivatives with respect to $T$ in the operators.

We rewrite Eq. (3) to express the output pulse in terms of the propagation operator applied to the input pulse, ${ }^{9}$

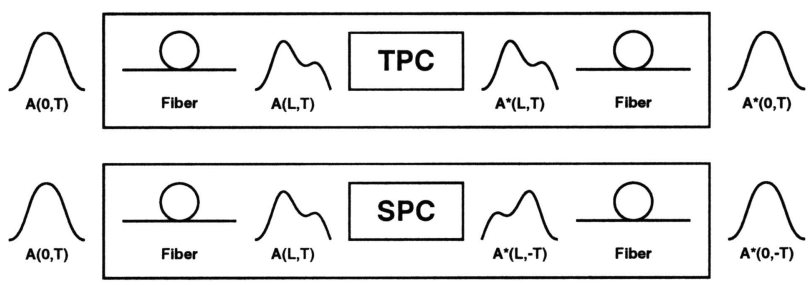

Fig. 1. Schematics of TPC and SPC. 


$$
\begin{aligned}
A(L, T)= & \exp \left\{L \hat{D}_{T}+\int_{0}^{L} \hat{N}_{T}[A(z, T)] \mathrm{d} z\right\} \\
& \times A(0, T),
\end{aligned}
$$

where $L$ is the fiber length. The input can also be expressed in terms of the output by applying the reverse propagation operator, ${ }^{7}$

$$
\begin{aligned}
A(0, T)= & \exp \left\{-L \hat{D}_{T}-\int_{0}^{L} \hat{N}_{T}[A(z, T)] \mathrm{d} z\right\} \\
& \times A(L, T) .
\end{aligned}
$$

Now let us take the complex conjugate of Eq. (7) and make the substitution $T \rightarrow-T$. Equation (7) becomes

$$
\begin{aligned}
A^{*}(0,-T)= & \exp \left\{-L \hat{D}_{-T}^{*}-\int_{0}^{L} \hat{N}_{-T}^{*}[A(z,-T)] \mathrm{d} z\right\} \\
& \times A^{*}(L,-T) .
\end{aligned}
$$

The conjugated and time-reversed linear operator, ignoring loss, is

$$
\begin{aligned}
\hat{D}_{-T}^{*} & =\sum_{n=2}^{\infty}-j \frac{\beta_{n}}{n !}\left[-j\left(-\frac{\partial}{\partial T}\right)\right]^{n} \\
& =\sum_{n=2}^{\infty}-j \frac{\beta_{n}}{n !}\left(j \frac{\partial}{\partial T}\right)^{n}=-\hat{D}_{T} .
\end{aligned}
$$

Similarly, the nonlinear operator, ignoring intrapulse Raman scattering, is

$$
\hat{N}_{-T}^{*}[A(z,-T)]=-\hat{N}_{T}\left[A^{*}(z,-T)\right] .
$$

In general, we only keep terms that acquire a minus sign when conjugation and time reversal are both applied. All operator terms, except loss and intrapulse Raman scattering, satisfy our criteria because of their odd combinations of $j$ 's and time derivatives. With the substitution $z \rightarrow L-z^{\prime}$ Eq. (8) becomes

$$
\begin{aligned}
A^{*}(0,-T)= & \exp \left\{L \hat{D}_{T}+\int_{0}^{L} \hat{N}_{T}\left[A^{*}\left(L-z^{\prime},-T\right)\right] \mathrm{d} z^{\prime}\right\} \\
& \times A^{*}(L,-T) .
\end{aligned}
$$

Equation (12) has the exact same form as Eq. (6) but with $A^{*}\left(L-z^{\prime},-T\right)$ as the solution. In other words, if we launch $A^{*}(L,-T)$ in another identical fiber, the final output $A^{*}(0,-T)$ is a conjugated and timereversed version of the first input. This result can be applied only to cases in which loss and intrapulse Raman scattering can be neglected. Table 1 summarizes the propagation effects that can be compensated for by TPC and SPC.

To identify important propagation effects for a given optical pulse transmission system, it is useful to define a characteristic length for each propagation effect ${ }^{9}$ :

$$
\begin{aligned}
L_{\text {loss }} & =\text { loss length }=1 / \alpha, \\
L_{D} & =\text { dispersion length }=T_{0}^{2} /\left|\beta_{2}\right|, \\
L_{D}^{\prime} & =\text { third-order dispersion length } \\
& =T_{0}^{3} /\left|\beta_{3}\right|, \\
L_{\mathrm{NL}} & =\text { nonlinear length }=1 / \gamma P_{0}, \\
L_{\mathrm{SS}} & =\text { self-steepening length }=\omega_{0} T_{0} / \gamma P_{0}, \\
L_{R} & =\text { Raman length }=T_{0} / T_{R} \gamma P_{0},
\end{aligned}
$$

where $T_{0}$ is the pulse width. The significance of a propagation effect can be roughly estimated by the ratio of the total propagation distance $L_{\text {total }}$ to the characteristic length. Hence a phase conjugation system should be designed such that the characteristic lengths of uncompensated propagation effects are much longer than $L_{\text {total }}$. This is demonstrated next in the numerical simulations.

As a numerical example, consider $\lambda_{0}=1550 \mathrm{~nm}$; two dispersion-shifted fibers, each with length $L_{\text {total }} / 2=$ $1 \mathrm{~km}$; parameters $\beta_{2}=-1 \mathrm{ps}^{2} / \mathrm{km}, \beta_{3}=0.1 \mathrm{ps}^{3} / \mathrm{km}$, $\gamma=1.5 \mathrm{~W}^{-1} \mathrm{~km}^{-1}, \alpha=0.2 \mathrm{~dB} / \mathrm{km}$, and $T_{R}=3 \mathrm{fs} ; \mathrm{a}$ temporal or spectral phase conjugator in the middle; an amplifier at each fiber end to compensate for loss; and a super-Gaussian input pulse,

$$
A(t)=\sqrt{P_{0}} \exp \left[-\frac{1}{2}\left(\frac{T}{T_{0}}\right)^{6}\right],
$$

with $T_{0}=200 \mathrm{fs}$ and peak power $P_{0}=1.7 \mathrm{~W}$. The peak power is chosen to be one tenth that of a fundamental soliton, such that $L_{\mathrm{loss}}=2 \mathrm{~km}, L_{D}=0.04 \mathrm{~km}$, $L_{D}^{\prime}=0.08 \mathrm{~km}$, and $L_{\mathrm{NL}}=0.4 \mathrm{~km}$. Other characteristic lengths are too long to be significant. Since $L_{\mathrm{NL}}$ is comparable with $L_{\text {total }}$ while much longer than the dispersion lengths, we expect nonlinear effects to be observable but less significant than dispersion effects. The output pulses with and without compensation schemes are plotted in Fig. 2. SPC reconstructs the input pulse at the output almost perfectly, whereas the TPC output pulse is significantly distorted by third-order dispersion. At this power level SPC has an advantage over TPC because of its ability to compensate for all important linear and nonlinear effects together with an amplifier.

In practice, SPC can be performed by spectral holography, ${ }^{4}$ short-pump four-wave mixing, ${ }^{10}$ or spectral

Table 1. Comparison of TPC and SPC in Terms of Propagation Effects That Can Be Compensated for by Each Scheme

\begin{tabular}{ccccccc}
\hline & Loss & $\mathrm{EOD}^{a}$ & $\mathrm{OOD}^{b}$ & $\mathrm{SPM}^{c}$ & $\mathrm{SS}^{d}$ & $\mathrm{IRS}^{e}$ \\
\hline TPC & $\times$ & $\sqrt{ }$ & $\times$ & $\sqrt{ }$ & $\times$ & $\sqrt{ }$ \\
SPC & $\times$ & $\sqrt{ }$ & $\sqrt{ }$ & $\sqrt{ }$ & $\sqrt{ }$ & $\times$ \\
\hline
\end{tabular}

${ }^{a}$ Even-order dispersion.

${ }^{b}$ Odd-order dispersion.

${ }^{c}$ Self-phase modulation.

${ }^{d}$ Self-steepening.

${ }^{e}$ Intrapulse Raman scattering. 


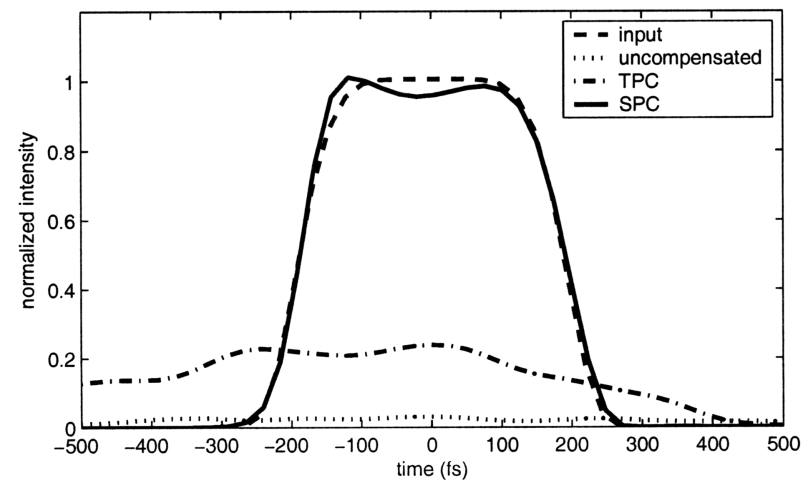

Fig. 2. Input and output pulses, with and without compensation schemes, when a $1.7-\mathrm{W} 200-\mathrm{fs}$ super-Gaussian pulse propagates for a total distance of $2 \mathrm{~km}$.

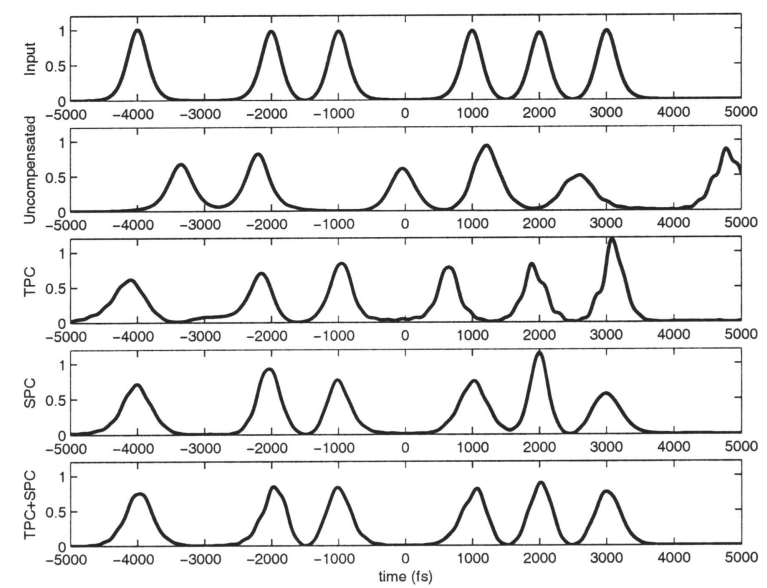

Fig. 3. Input and output pulses, with and without compensation schemes, when multiple $200-\mathrm{fs}$ solitons propagate for a total distance of $1 \mathrm{~km}$.

four-wave mixing. ${ }^{11}$ If SPC is to be used in a communication system, one must perform time reversal only on each time slot or a group of slots, within the time window of the SPC device. A synchronous clock, in the form of pump pulses, is therefore unfortunately required. The pulses also need to be periodically conjugated before they breach adjacent time windows. In this case solitons are preferred because their broadening is much slower than that of conventional pulses and the frequency of conjugation can be minimized. We note that periodic conjugation is also required in other schemes for different reasons, such as that suggested by Pina et al., ${ }^{8}$ to satisfy the path-averaging assumption.

Since SPC can compensate for distortions that TPC cannot compensate for and vice versa, we propose that a hybrid scheme combining SPC and TPC can offer superior performance. An example would be to sandwich a temporal phase conjugator with two midway SPC systems such that the Raman effect uncompensated in a SPC system can be compensated for by the TPC system, at least to first order. More rigorous analysis is required to fully estimate the performance of a hybrid scheme.

Our second numerical example tests the compensation capabilities of SPC and the hybrid scheme for multiple solitons. It has been suggested that TPC can compensate for soliton interactions. ${ }^{12}$ Figure 3 plots the output pulses obtained from various compensation schemes for the same parameters as the first example but with a total length of $1 \mathrm{~km}$ and a $17-\mathrm{W}$ alternatively $\pi$ phase-shifted sech soliton train representing the bit sequence 10110111. SPC undoes soliton interactions and pulse distortions better than TPC in this case, whereas the hybrid scheme performs slightly better than SPC. This can be attributed to the fact that the hybrid scheme has more phase conjugation stages for the same total length. The hybrid scheme, however, can also compensate for the Raman-induced frequency shift, which cannot be compensated by SPC alone. The mean frequency shift of the TPC output is calculated to be $+0.032 \mathrm{THz}$, that of the SPC output is $-0.16 \mathrm{THz}$, and that of the hybrid scheme is only $-0.027 \mathrm{THz}$.

In summary, we have proved that SPC can compensate for all the linear and nonlinear distortions to optical pulses considered if loss and intrapulse Raman scattering can be neglected. Moreover, SPC and a hybrid scheme combining TPC and SPC are both shown numerically to offer better compensation of pulse distortions and soliton interactions than TPC for femtosecond pulses.

We thank F. G. Omenetto of Los Alamos National Laboratory for inspiring discussions. This work was supported primarily by the Engineering Research Centers Program of the National Science Foundation under award EEC-9402726. M. Tsang's e-mail address is mankei@sunoptics.caltech.edu.

\section{References}

1. A. Yariv, D. Fekete, and D. M. Pepper, Opt. Lett. 4, 52 (1979).

2. R. A. Fisher, B. R. Suydam, and D. Yevick, Opt. Lett. 8, 611 (1983).

3. S. Chi and S. F. Wen, Opt. Lett. 19, 1705 (1994).

4. A. M. Weiner, D. E. Leaird, D. H. Reitze, and G. Paek, IEEE J. Quantum Electron. 28, 2251 (1992).

5. C. Chang, H. P. Sardesai, and A. M. Weiner, Opt. Lett. 23, 283 (1998).

6. F. G. Omenetto, A. J. Taylor, M. D. Moores, and D. H. Reitze, Opt. Lett. 26, 938 (2001).

7. M. Tsang, D. Psaltis, and F. G. Omenetto, Opt. Lett. (to be published).

8. J. Pina, B. Abueva, and G. Goedde, Opt. Commun. 176, 397 (2000).

9. G. P. Agrawal, Nonlinear Fiber Optics (Academic, San Diego, Calif., 2001).

10. D. A. B. Miller, Opt. Lett. 5, 300 (1980).

11. D. Marom, D. Panasenko, R. Rokitski, P. Sun, and Y. Fainman, Opt. Lett. 25, 132 (2000).

12. W. Forysiak and N. J. Doran, Electron. Lett. 30, 154 (1994). 